\title{
LincRNA-p21 enhances the sensitivity of radiotherapy for human colorectal cancer by targeting the Wnt/ $\beta$-catenin signaling pathway
}

\author{
GUANGYU WANG $^{1}$, ZHIWEI LI $^{1}$, QI ZHAO ${ }^{1}$, YUANYUAN ZHU ${ }^{1}, \mathrm{CI} \mathrm{ZHAO}^{1}$, \\ $\mathrm{XIN} \mathrm{LI}^{1}, \mathrm{ZHIGANG} \mathrm{MA}^{1},{\mathrm{XIAOBO} \mathrm{LI}^{2} \text { and YANQIAO ZHANG }}^{1}$
}

${ }^{1}$ Department of Gastrointestinal Medical Oncology, The Affiliated Tumor Hospital of Harbin Medical University, Harbin, Heilongjiang 150040; ${ }^{2}$ Department of Pathology, Harbin Medical University, Harbin, Heilongjiang 150081, P.R. China

Received December 15, 2013; Accepted February 11, 2014

DOI: 10.3892/or.2014.3047

\begin{abstract}
Recent studies show that long intergenic noncoding RNA-p21 (lincRNA-p21) is aberrantly expressed in several types of cancer, including colorectal cancer (CRC), one of the most common cancers in the world. Radiotherapy is considered as a standard preoperative treatment approach to reduce local recurrence for local advanced rectal cancer. However, a considerable number of rectal cancers are resistant to radiotherapy. In the present study, we evaluated the role of lincRNA-p21 in radiotherapy for CRC and detected the possible molecular mechanism. By expression profile analysis, we demonstrated that lincRNA-p21 decreases in CRC cell lines and tissue samples, which contributes to the elevation of $\beta$-catenin in CRC. We further showed that lincRNA-p21 increases following X-ray treatment, and enforced expression of the lincRNA enhances the sensitivity of radiotherapy for CRC by promoting cell apoptosis. Suppression of the $\beta$-catenin signaling pathway and elevation of the pro-apoptosis gene Noxa expression may help explain the role of lincRNA-p21 in CRC radiotherapy. The present study not only deepens our understanding of the mechanism of radiotherapy for CRC, but it also provides a potential target for CRC radiotherapy.
\end{abstract}

\section{Introduction}

Long intergenic noncoding RNAs (lincRNAs) are non-protein coding transcripts $>200$ nucleotides in length. They have been identified for decades, but they failed to draw attention until now, mainly due to the limitation of technology and traditional conceptual understanding of molecular biology. LincRNAs are

Correspondence to: Professor Yanqiao Zhang, Department of Gastrointestinal Medical Oncology, The Affiliated Tumor Hospital of Harbin Medical University, 150 Haping Road, Nangang, Harbin, Heilongjiang 150040, P.R. China

E-mail: yanqiaozhang@ems.hrbmu.edu.cn

Key words: lincRNA-p21, colorectal cancer, Wnt/ $\beta$-catenin signaling pathway, cell apoptosis, radiosensitivity located and transcribed within the intergenic stretches with the exception that a minority of them are within protein coding genes; their length varies from several hundred to tens of thousands of bases $(1,2)$. Large scale transcriptomic sequencing by next generation sequencing indicated that lincRNA number in the order of tens of thousands in mammals. However, despite accumulating evidence suggesting that the majority of these are likely to be functional, only a relatively small proportion has been functionally annotated (3). From collected data, lincRNAs regulate target gene expression at both the transcriptional and post-transcriptional level. LincRNAs regulate gene transcription through either targeting specific transcriptional factors or targeting the general transcriptional machinery (4). LincRNAs also regulate the transcription of large numbers of genes by chromatin modifications (5). In addition to regulating transcription, lincRNAs also control various aspects of post-transcriptional mRNA processing, including splicing, transport, translation and degradation of target mRNAs $(6,7)$. LincRNAs not only play critical roles in physical function regulation, such as imprinting (8), but they are also involved in the progress of various diseases including cancer (9-11). At present, lincRNAs are emerging as either oncogenes or tumor suppressor genes (11-15). Previous evidence demonstrated the aberrant expression of lincRNAs in digestive system carcinomas (16-21). However, the biological function of lincRNAs in the vast majority of digestive system carcinomas remains unclear. LincRNA-p21 has been found to be a downstream target of p53 and to modulate the expression of numerous genes at the transcriptional level (22). The expression of lincRNA-p21 is found to be downregulated in several types of tumor $(22,23)$, suggesting that lincRNA-p21 may function as a tumor suppressor. Recently, lincRNAp21 was found to inhibit the expression of $\beta$-catenin at the post-transcriptional level (24). It has been reported that the elevation of the Wnt $/ \beta$-catenin signaling pathway is one of the common features of colorectal cancer (CRC) (25). Recent studies also demonstrated that $\mathrm{Wnt} / \beta$-catenin signaling is one of the effective targets for chemotherapy and chemoprevention of CRC (26-28). A recent study showed that expression of lincRNA-p21 was lower in human CRC tissue compared to paired normal tissue (23). However, the pathological role 
of decreased lincRNA-p21 in CRC was not detected. Since lincRNA-p21 has been reported to inhibit the translation of $\beta$-catenin in HeLa cells (24), and $\beta$-catenin aberrant activation affected CRC treatment, we hypothesized that lincRNA-p21 may affect the treatment of $\mathrm{CRC}$ through regulating $\beta$-catenin activity. To test this hypothesis, we detected the expressional change of lincRNA-p21 and evaluated the role of lincRNA-p21 in CRC radiotherapy; the regulation role of lincRNA-p21 in the Wnt/ $\beta$-catenin signaling pathway in CRC cells as a candidate for the molecular mechanism of lincRNA-p21 regulating the radiosensitivity of $\mathrm{CRC}$ radiotherapy was also detected.

\section{Materials and methods}

Patient samples. The present study consisted of 30 CRC tissues and their adjacent normal mucosa, which were resected at the Department of Gastrointestinal Surgical Oncology (The Affiliated Tumor Hospital of Harbin Medical University) between 2011 and 2012. None of the patients received preoperative treatment such as irradiation or chemotherapy. All research protocols in the present study were approved by the Ethics Committee of the Cancer Research Institute at Harbin Medical University. Staging of the tumors was performed according to NCCN Guidelines.

Cell lines and cell culture. The normal colorectal cell line, FHC, and the CRC cell lines SW1116, SW620, LS 174T, HT29 and LOVO were provided by the Shanghai Institute of Cell Biology (Shanghai, China). The cell lines were maintained in Dulbecco's modified Eagle's medium or RPMI-1640, respectively, containing $10 \%$ FBS with $100 \mathrm{U} / \mathrm{ml}$ penicillin and $100 \mu \mathrm{g} / \mathrm{ml}$ streptomycin, and were cultured in a humidified $5 \% \mathrm{CO}_{2}$ incubator at $37^{\circ} \mathrm{C}$. The medium was changed every 2 days (29). Exponentially growing cells were used for experiments.

Irradiation treatment of cells. The $3 \times 10^{5}$ cells were plated in triplicate in $25 \mathrm{~cm}^{2}$ tissue culture dishes. After transfecting for $24 \mathrm{~h}$, cells were irradiated with various single radiation doses ( 0,2 and $4 \mathrm{~Gy}) \mathrm{X}$-ray. Then, cells were harvested at $0,0.5,1,2$, $6,12,24$ and $48 \mathrm{~h}$ after irradiation for further analyses.

Total RNA preparation and reverse transcription. Total RNA from colorectal and CRC epithelial cells and tissues was extracted using TRIzol as previously described (30). Agarose gel electrophoresis identified integrity of total RNA. The ratio of A260:A280 was used to indicate the purity of total RNA. cDNA was generated using the First Strand cDNA Synthesis kit (Roche), according to the manufacturer's instructions.

Quantitative real-time polymerase chain reaction ( $q R T-P C R)$ assay. To detect and compare gene expression, qRT-PCR was performed with a Power SYBR-Green PCR Master Mix (Roche, Switzerland) and an Applied Biosystems 7500 Fast System (ABI; Foster City, CA, USA) as described by Wang et al (31). Relative levels of gene expression were determined with glyceraldehyde-3-phosphate dehydrogenase (GAPDH) as the control. The cycle number at which the reaction crossed an arbitrarily placed threshold $(\mathrm{Ct})$ was determined for each gene and the $\Delta \Delta \mathrm{Ct}$ method $\left(2^{-\Delta \mathrm{Ct}}\right)$ was used to determine the target
Table I. The primer sequences used in the present study.

\begin{tabular}{ll}
\hline Name & \multicolumn{1}{c}{ Sequence } \\
\hline LincRNA-p21 & F: GGGTGGCTCACTCTTCTGGC \\
LincRNA-p21 & R: TGGCCTTGCCCGGGCTTGTC \\
$\beta$-Catenin & F: ATTGTCCACGCTGGATTTTC \\
$\beta$-Catenin & R: TCGAGGACGGTCGGACT \\
GAPDH & F: AGCCACATCGCTCAGACAC \\
GAPDH & R: GCCCAATACGACCAAATCC \\
Noxa & F: ATGAATGCACCTTCACATTCCTCT \\
Noxa & R:TCCAGCAGAGCTGGAAGTCGAGTGT \\
c-Myc & F: CGCTTCTCTGAAAGGCTCTCCTTG \\
c-Myc & R: GAGTCGTAGTCGAGGTCATAGTTC \\
Cyclin D1 & F: AGGAGAACAAACAGATCA \\
Cyclin D1 & R: TAGGACAGGAAGTTGTTG \\
TCF4 & F: GAGAAT-TCATGCCGCAGCT \\
TCF4 & R: CAGATATCTTTTTAAACGCTACA
\end{tabular}

gene levels relative to those of GAPDH. The primers used in this study are summarized in Table I.

Western blot assay. Total protein was extracted using SDS protein lysis buffer. Protein $(80 \mu \mathrm{g})$ for each sample was resolved by $8 \%$ SDS-PAGE and then transferred to PVDF membranes. The membranes were blocked by TBST buffer (TBS plus $0.1 \%$ Tween-20) containing 5\% w/v skimmed milk and hybridized with primary antibody including rabbit anti$\beta$-catenin (1:500), rabbit anti-c-myc (1:750; both from Abcom, Hong Kong), mouse anti- $\beta$-actin (1:5,000; Santa Cruz), rabbit anti-cyclin D1 (1:50; Abcom), followed by incubation with specific HRP-conjugated secondary antibody (ZSGB-BIO, Beijing). Protein bands were visualized by the ECL detecting system (Applygen, Beijing).

RNA interference and overexpression. RNA interference for lincRNA-p21 was performed as previously described (22). Several siRNA oligos targeting lincRNA-p21 (\#1 UGA AAAGAGCCGUGAGCUA, \#2 AAAUAAAGAUGGUGGA AUG and \#3 AGUCAAAGGCAAUGAGCAU) and Lipofectamine 2000 were purchased from Invitrogen. The siRNA transfections were performed with $50 \mathrm{nM}$ of siRNA and Lipofectamine 2000 in serum-free culture media following the manufacturer's instructions as previously described $(32,33)$. A plasmid expressing lincRNA-p21 was used to construct plasmid plincRNA-p21pcDNA3.1 (+). The plasmid was previously reported (24) and kindly provided by Prof. Myriam Gorospe.

Analysis of cell apoptosis. Cells undergoing early and late apoptosis were quantified using flow cytometry analysis (Cytomics FC500; Beckman Coulter, Miami, FL, USA), following the staining with Annexin V-FITC and propidium iodide (PI; BD Biosciences, San Jose, CA, USA). All experiments were performed in triplicate.

Statistical analysis. Statistical analysis was performed with SPSS13.0 software. Results are expressed as the means \pm stan- 
A

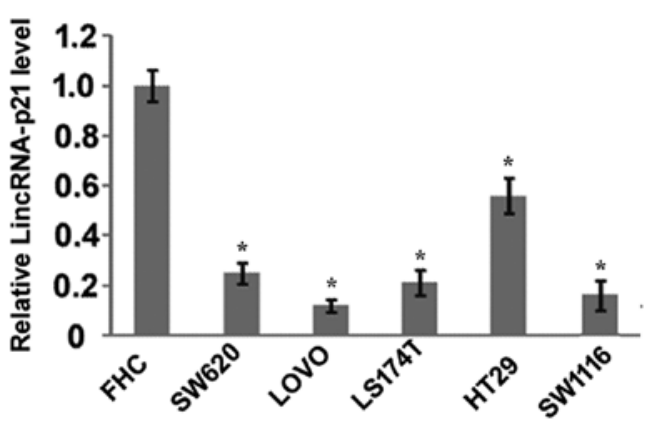

B

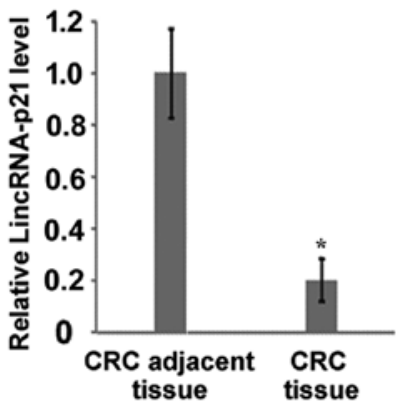

Figure 1. LincRNA-p21 expression level in CRC cell lines and CRC tumor tissues. (A) The expression of lincRNA-p21 in all the tested CRC cell lines (SW620, LOVO, LS174T, HT29 and SW116) was lower than in the norma colorectal cell line, FHC. (B) The expression of lincRNA-p21 in CRC tissues was significantly decreased compared with their adjacent tissues. Values represent means $\pm \mathrm{SD}$ from at least 3 independent experiments. ${ }^{*} \mathrm{P}<0.05$. dard deviation from at least 3 separate experiments. Standard statistical tests including Student's t-test and one-way ANOVA were used to evaluate the significance. $\mathrm{P}<0.05$ was considered to indicate a statistically significant difference.

\section{Results}

LincRNA-p21 is downregulated in CRC cell lines and CRC tumor tissues. To examine the potential roles of lincRNA-p21 in CRC, we first compared the expression of lincRNA-p21 between normal colorectal epithelial cells and CRC cell lines. Our results showed that the expression of lincRNA-p21 in all the tested CRC cell lines (SW620, LOVO, LS174T, HT29 and SW116) was lower than in the normal colorectal cell line FHC (Fig. 1A). Furthermore, we compared the expression of lincRNA-p21 in $30 \mathrm{CRC}$ tissues and their paired adjacent tissues. The results demonstrated that the expression of lincRNA-p21 in CRC tissues was significantly decreased compared with their adjacent tissues (Fig. 1B). These results suggest that lincRNA-p21 is downregulated in CRC, which may contribute to the development of CRC and/or affect the treatment of CRC.

LincRNA-p21 enhances radiosensitivity by promoting apoptosis. Radiotherapy is considered as a standard preoperative treatment approach to reduce local recurrence for locally advanced rectal cancers (34-37). To detect the potential role of lincRNA-p21 in CRC radiotherapy, we first detected the effect
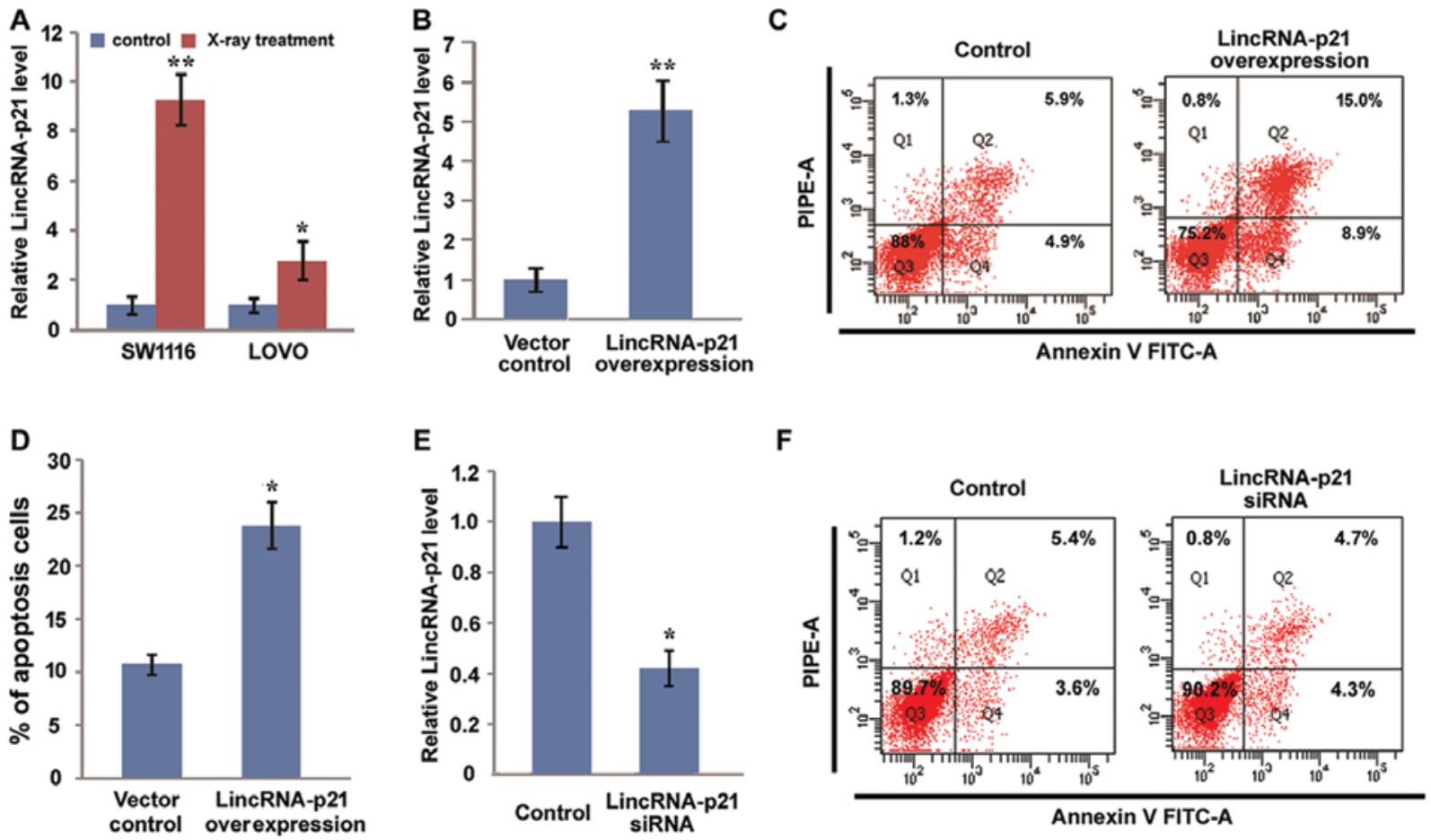

Figure 2. LincRNA-p21 enhances radiosensitivity by promoting apoptosis. (A) X-ray (4 Gy) treatment elevated the expression of lincRNA-p21 in both SW1116 and LOVO cells. Values represent means \pm SD from at least 3 independent experiments. ${ }^{* *} \mathrm{P}<0.01$ vs. untreated. (B) LincRNA-p 21 was overexpressed in SW1116 cells. Values represent means \pm SD from at least 3 independent experiments. ${ }^{* *} \mathrm{P}<0.01$ vs. control vector. (C) LincRNA-p21 overexpressed cells and control cells were treated with X-ray. Subsequently, the apoptosis of both group cells was detected with flow cytometry. (D) The apoptosis rate of lincRNA-p21 overexpressed cells was $\sim 25 \%$, while it was only $10 \%$ in control cells. Values represent means \pm SD from at least 3 independent experiments. ${ }^{*} P<0.05$ vs. control vector. (E) LincRNA-p21 was successfully inhibited by siRNA in SW1116 cells. Values represent means \pm SD from at least 3 independent experiments. ${ }^{*} \mathrm{P}<0.05$ vs. control siRNA. (F) There was no significant difference in the apoptosis rate between lincRNA-p21 knockdown cells and control cells. 
A

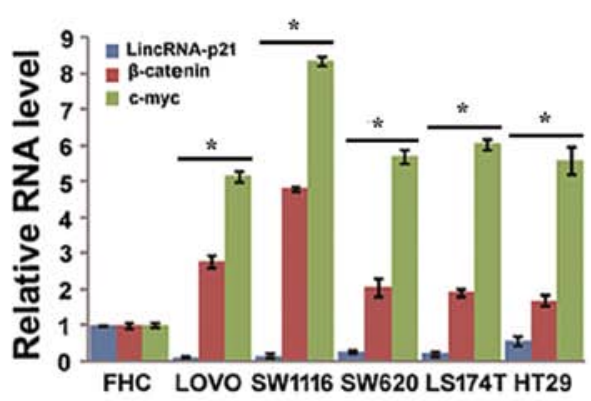

C

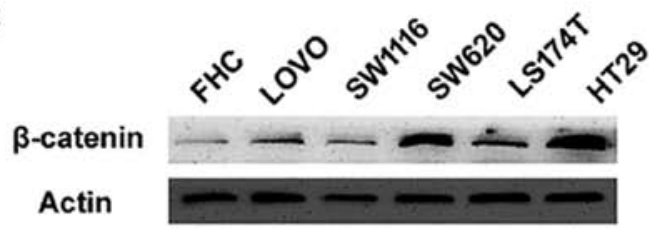

E

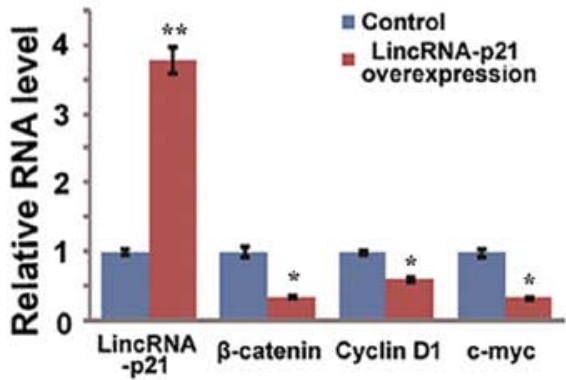

G

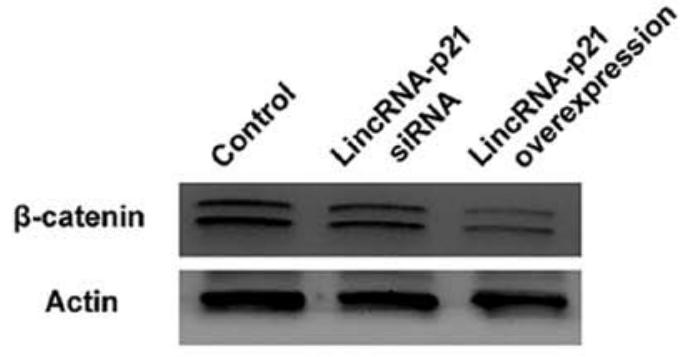

B

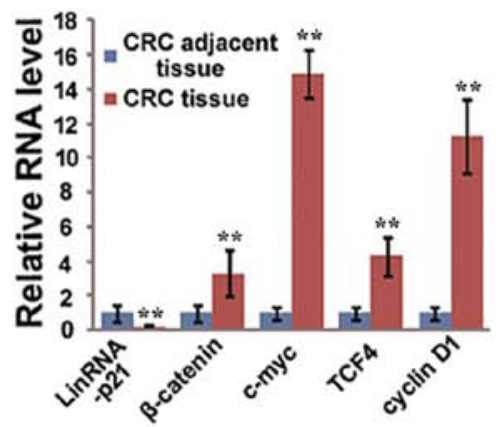

D
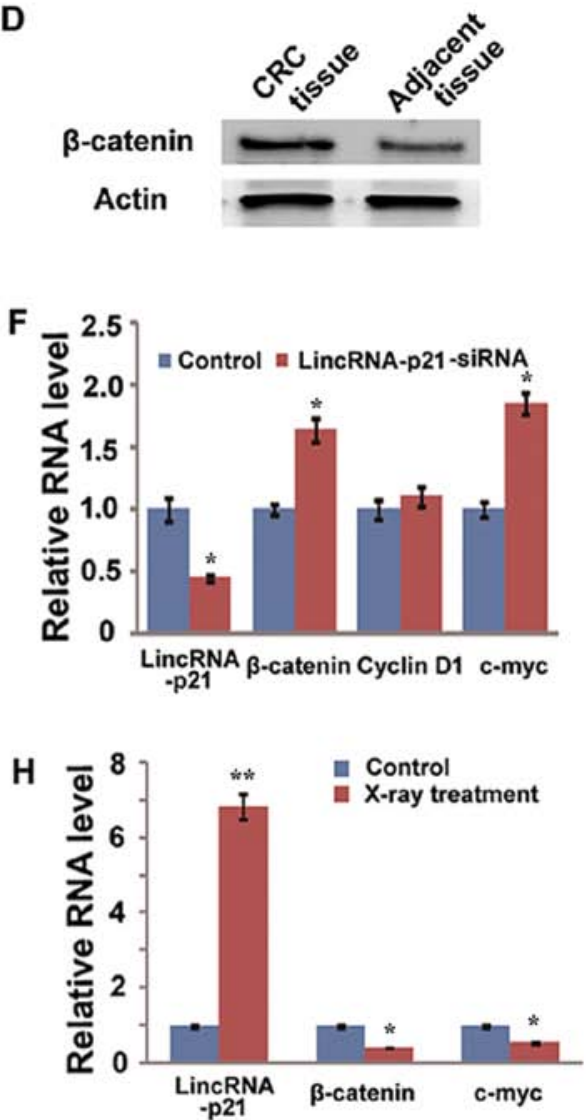

Figure 3. LincRNA-p21 inhibits the Wnt/ $\beta$-catenin signaling pathway. (A) The mRNA expression levels of lincRNA-p21, $\beta$-catenin and c-myc were detected using qRT-PCR in all the tested CRC cell lines and FHC cells. Values represent means \pm SD from at least 3 independent experiments. ${ }^{*} \mathrm{P}<0.05$ vs. FHC. (B) LincRNA-p21, $\beta$-catenin c-myc, cyclin D1 and TCF4 were detected using qRT-PCR in CRC tissues and adjacent tissues. Values represent means \pm SD from at least 3 independent experiments. ${ }^{* *} \mathrm{P}<0.01$ vs. control adjacent tissues. (C) The protein expression level of $\beta$-catenin was higher in all the tested CRC cell lines compared with FHC cells. (D) The protein expression level of $\beta$-catenin was higher in CRC tissues compared with adjacent tissues. (E) The overexpression of lincRNA-p21 inhibited the expression of $\beta$-catenin, cyclin D1 and c-myc. Values represent means \pm SD from at least 3 independent experiments. "P<0.05; ${ }^{* *} \mathrm{P}<0.01$ vs. control vector. (F) The knockdown of lincRNA-p21 regulated the expression of $\beta$-catenin, cyclin D1 and c-myc. Values represent means \pm SD from at least 3 independent experiments. ${ }^{*} \mathrm{P}<0.05$ vs. control siRNA. (G) The overexpression of lincRNA-p21 inhibited the protein expression level of $\beta$-catenin. (H) The expression of lincRNA-p21 was increased, while the expression of $\beta$-catenin and c-myc were decreased in SW1116 cells after X-ray treatment. Values represent means $\pm \mathrm{SD}$ from at least 3 independent experiments. ${ }^{*} \mathrm{P}<0.05 ;{ }^{* * *} \mathrm{P}<0.01$ vs. untreated.

of radiotherapy on the expression of lincRNA-p21. We showed that X-ray treatment elevated the expression of lincRNA-p21 in both SW1116 and LOVO cells (Fig. 2A). Then, we overexpressed lincRNA-p21 in SW1116 cells (Fig. 2B) and treated lincRNA-p21 overexpressed cells and control cells with X-ray. Subsequently, the apoptosis of both group cells was detected with Annexin V and PI staining by fluorescence activated cell sorting (FACS). Our results showed that the apoptosis rate of lincRNA-p21 overexpressed cells was $\sim 25 \%$, while it was only $10 \%$ in control cells (Fig. 2C and D), which suggested that overexpression of lincRNA-p21 increases the sensitivity of CRC radiotherapy by promoting the apoptosis of CRC cells. Furthermore, we inhibited endogenous lincRNA-p21 expression by using siRNA. To avoid the off-target effect of siRNA, we transfected SW1116 cells with a mixture of three synthesized lincRNA-p21 siRNAs; the results showed endogenous lincRNA-p21 decreased $60 \%$ after transfection with lincRNA-p21 siRNAs, which suggests that lincRNA-p21 was successfully inhibited by siRNA in SW1116 cells (Fig. 2E). Then, we evaluated the effect of lincRNA-p21 knockdown on 
A

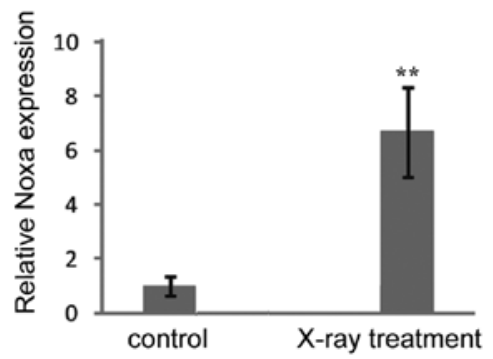

B

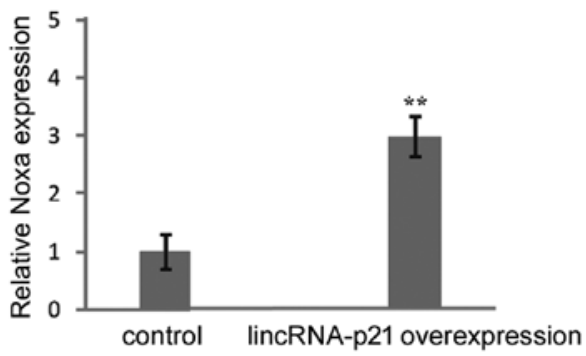

Figure 4. Both X-ray irradiation and lincRNA-p21 affect the expression of Noxa. (A) X-ray (4 Gy) treatment resulted in the upregulation of Noxa expression in SW1116 cells. Values represent means \pm SD from at least 3 independent experiments. ${ }^{* *} \mathrm{P}<0.01$ vs. untreated. (B) The overexpression of lincRNA-p21 led to an increase of Noxa expression. Values represent means $\pm \mathrm{SD}$ from at least 3 independent experiments. ${ }^{* *} \mathrm{P}<0.01$ vs. control vector.

the apoptosis of SW1116 cells induced by X-ray irradiation. We did not observe a significant difference in the apoptosis rate between lincRNA-p21 knockdown cells and control cells. Our results indicate that the expression level of lincRNA-p21 in CRC may affect the sensitivity of CRC treatment.

LincRNA-p21 inhibits the Wnt/ $\beta$-catenin signaling pathway. LincRNA-p21 has been reported to inhibit the translation of $\beta$-catenin in HeLa cells (24); however, whether lincRNA-p21 also inhibits the translation of $\beta$-catenin in CRC remains unknown. We first detected the relationship between lincRNA-p21 and the Wnt/ $\beta$-catenin signaling pathway in normal colorectal epithelial cells and CRC cell lines. Our results showed that $\beta$-catenin was highly expressed in all the tested CRC cell lines, at both the mRNA and the protein level, compared with FHC cells (Fig. 3A and C). LincRNA-p21 was lower in these CRC cell lines than in the FHC cells (Fig. 3A). Furthermore, the activity of $\mathrm{Wnt} / \beta$-catenin signaling pathway was investigated in these cell lines by detecting expression of c-myc, one of the target genes of $\beta$-catenin, and the results showed that c-myc level was higher in all the CRC cell lines than in the FHC cells (Fig. 3A). The relationship between lincRNA-p21 and $\beta$-catenin was also detected in CRC tissues and CRC adjacent tissues. The total RNA of $30 \mathrm{CRC}$ tissues and 30 adjacent tissues were mixed together, respectively. Then, the expression of lincRNA-p21, $\beta$-catenin and $\mathrm{Wnt} / \beta$-catenin target genes including c-myc, cyclin D1 and TCF4 was detected using qRT-PCR. The results showed that $\beta$-catenin and its target genes were significantly increased in CRC tissues when compared with CRC adjacent tissues (Fig. 3B). Consistently, the protein level of $\beta$-catenin was also confirmed to be elevated in CRC tissues (Fig. 3D). These results suggest that there is an inverse correlation between the expression of lincRNA-p21 and the expression and activity of $\beta$-catenin in the CRC cell lines and tissues, and it suggests that lincRNA-p21 may regulate the expression and activity of $\beta$-catenin in CRC.

We further detected the direct impact of lincRNA-p21 on the expression of $\beta$-catenin by gain-of-function and lossof-function strategy, respectively. We demonstrated that lincRNA-p21 overexpression in SW1116 cells significantly inhibited the expression of $\beta$-catenin at both the mRNA and the protein level (Fig. 3E and G). Furthermore, the overexpression of lincRNA-p21 inhibited Wnt/ $\beta$-catenin signaling activity as evidenced by the decrease of $\beta$-catenin target genes, such as cyclin D1 and c-myc (Fig. 3E). However, the knockdown of
lincRNA-p21 failed to promote the expression of $\beta$-catenin at both the mRNA and the protein level (Fig. 3F and G). The obvious activation of $\mathrm{Wnt} / \beta$-catenin signaling pathway caused by the knockdown of lincRNA-p21 was also not observed (Fig. 3F).

Finally, to investigate whether the inhibition of $\mathrm{Wnt} / \beta$ catenin is involved in the mechanism of lincRNA-p21 enhanced sensitivity of CRC radiotherapy, we detected the expression and activity of $\beta$-catenin in SW1116 cells after X-ray treatment. We showed that the irradiation with X-ray decreased the expression and activation of $\beta$-catenin (Fig. $3 \mathrm{H}$ ). This result suggests that the inhibition of $\beta$-catenin is involved in the lincRNA-p21 regulating sensitivity of CRC radiotherapy.

LincRNA-p21 promotes pro-apoptosis gene Noxa expression. Noxa, a pro-apoptosis gene, is positively regulated by lincRNAp21 and contributes to cell apoptosis after p53 activation (22). We detected the effect of X-ray irradiation and lincRNA-p21 on the expression of Noxa. We showed that X-ray treatment resulted in the upregulation of Noxa expression in SW1116 cells (Fig. 4A). The overexpression of lincRNA-p21 also led to increase of Noxa expression (Fig. 4B). These results suggested that the upregulated pro-apoptosis gene Noxa resulting from lincRNA-p21 elevation after X-ray irradiation may also be involved in the molecular mechanism of lincRNA-p21 enhancing sensitivity of CRC radiotherapy.

\section{Discussion}

Recent studies have shown that lincRNA-p21 levels may significantly influence the biological properties of cells, including tumor cell proliferation and apoptosis $(22,38)$. CRC is the third most common form of cancer and the fourth most frequent cause of cancer-related mortality, with $>1$ million new cases each year worldwide (39). However, current knowledge about lincRNA-p21 function in human CRC is still preliminary. Therefore, determining whether lincRNAp21 is essential for the progression of CRC may provide a promising therapeutic target. Our findings showed that the expression level of lincRNA-p21 was significantly lower in $\mathrm{CRC}$ tissue compared with the adjacent normal tissue from the same patient. It is well known that $\sim 90 \%$ of CRC cases originate from the constitutive activation of $\mathrm{Wnt} / \beta$-catenin signaling pathway. Previous reports demonstrated that the Wnt/ $\beta$-catenin signaling pathway is a crucial pathway in the 
initiation and progression of CRC (37,40-42). Disruption of the Wnt/ $\beta$-catenin signaling pathway caused by either mutant Wnt or stabilization of the $\beta$-catenin protein resulted in activation of the TCF-4 transcription factor and increased expression of target genes including c-myc and cyclin D1 (43). The c-myc and cyclin D1 are identified as target genes in the Wnt/ $\beta$-catenin signaling pathway and overexpress in CRC $(44,45)$. In the present study, we showed that lincRNA-p21 was inversely correlated with $\mathrm{Wnt} / \beta$-catenin signaling pathway target genes in both clinical tissue specimens and human CRC cell lines, and the overexpression of lincRNA-p21 significantly inhibited Wnt/ $\beta$-catenin signaling activity through directly inhibiting $\beta$-catenin stability and/or translation. Our results are consistent with recent findings that $\beta$-catenin translation is suppressed by lincRNA-p21 in human HeLa cell line (24). However, obvious activation of the Wnt/ $\beta$-catenin signaling pathway caused by the knockdown of lincRNA-p21 was not observed in this study. Our results showed that lincRNA-p21 level was very low in CRC cell lines, thus we speculate that these models are not the most suitable for knockdown models in this setting. This could not exclude that lincRNA-p21 may be a necessary but not sufficient factor for $\beta$-catenin inhibition.

Radiotherapy is considered as a standard preoperative treatment approach to reduce local recurrence for locally advanced rectal cancers $(34,35)$. However, considerable rectal cancers are resistant to preoperative radiotherapy (46) and radiotherapy may result in strong side-effects in some patients. Therefore, increasing radiosensitivity may be very useful for enhancing the effect of therapy while reducing the side-effects of X-ray irradiation. A previous study showed that anti-vascular endothelial growth factor therapy may enhance radiosensitization in patients with locally advanced inoperable CRC (36). Radiosensitization may occur through a direct effect on CRC cells followed by suppression of some transcription factors or genes (37). Whether the transcription factor $\beta$-catenin is one of the targets for CRC radiotherapy and if lincRNA-p21 affects radiosensitivity is unknown. Our results showed that $\beta$-catenin is decreased while lincRNA-p21 is increased after X-ray irradiation, and the enforced expression of lincRNA-p21 decreases the surviving fraction of tumor cells in response to $\mathrm{X}$-ray irradiation and enhances radiosensitivity. We further showed that lincRNA-p21 is likely to enhance radiosensitivity by inducing tumor cell apoptosis. However, there is no significant change of cell apoptosis after X-ray irradiation in cells treated with lincRNA-p21 siRNA compared with those treated with negative siRNA control. This result is consistent with the expression change of $\beta$-catenin following lincRNA-p21 knockdown.

Pro-apoptotic protein Noxa initiates apoptosis by binding to regulatory sites on anti-apoptotic Bcl-2 protein and directly neutralizing its function. A previous study showed that a chemotherapy drug may induce the pro-apoptotic protein Noxa in CRC cells (47). Other studies indicated that the loss of Noxa may enhance resistance to X-ray irradiation-induced apoptosis $(48,49)$. Consistent with these previous studies, our results showed that X-ray treatment may activate lincRNA-p21 and Noxa expression and then induce CRC cell apoptosis. Moreover, we found that lincRNA-p21 can promote cell apoptosis after X-ray irradiation by increasing Noxa expression. Our data provide an interesting link between lincRNA-p21 and Noxa, and suggest that Noxa acts as a downstream target of lincRNA-p21. This result is consistent with a previous report in which the expression of Noxa was found to change according to lincRNA-p21 status identified by gene expression microarray (22).

In conclusion, we first found that lincRNA-p21 increases the sensitivity of radiotherapy for CRC by targeting the $\beta$-catenin signaling pathway. The present study not only deepens our understanding of the mechanism of radiotherapy for CRC, but it also provides a potential target for CRC radiotherapy. Our results also suggest that lincRNA-p21 itself and compounds that can induce the expression of lincRNA-p21 may be useful for enhancing the sensitivity of CRC radiotherapy.

\section{Acknowledgements}

The authors thank Professor Myriam Gorospe of the National Institutes of Health Laboratory of Molecular Biology and Immunology for kindly providing lincRNA-p21 expression plasmid. The authors also acknowledge the technical assistance of Yuyan Ma and Yanmei Yang of the Cancer Research Institute of Harbin Medical University. This study was supported by the National Natural Science Foundation of China (grant no. 81172265).

\section{References}

1. Guttman M, Amit I, Garber M, et al: Chromatin signature reveals over a thousand highly conserved large non-coding RNAs in mammals. Nature 458: 223-227, 2009.

2. Katayama S, Tomaru Y, Kasukawa T, et al: Antisense transcription in the mammalian transcriptome. Science 309: 1564-1566, 2005.

3. Amaral PP, Clark MB, Gascoigne DK, Dinger ME and Mattick JS: lncRNAdb: a reference database for long noncoding RNAs. Nucleic Acids Res 39: D146-D151, 2011.

4. Goodrich JA and Kugel JF: Non-coding-RNA regulators of RNA polymerase II transcription. Nat Rev Mol Cell Biol 7: 612-616, 2006.

5. Chen X, Xu H, Yuan P, et al: Integration of external signaling pathways with the core transcriptional network in embryonic stem cells. Cell 133: 1106-1117, 2008.

6. Beltran M, Puig I, Peña C, et al: A natural antisense transcript regulates Zeb2/Sip1 gene expression during Snail1-induced epithelial-mesenchymal transition. Genes Dev 22: 756-769, 2008.

7. Centonze D, Rossi S, Napoli I, et al: The brain cytoplasmic RNA $\mathrm{BC} 1$ regulates dopamine $\mathrm{D}_{2}$ receptor-mediated transmission in the striatum. J Neurosci 27: 8885-8892, 2007.

8. Braidotti G, Baubec T, Pauler F, et al: The Air noncoding RNA: an imprinted cis-silencing transcript. Cold Spring Harb Symp Quant Biol 69: 55-66, 2004.

9. Fu X, Ravindranath L, Tran N, Petrovics G and Srivastava S: Regulation of apoptosis by a prostate-specific and prostate cancer-associated noncoding gene, PCGEM1. DNA Cell Biol 25: 135-141, 2006.

10. Lin R, Maeda S, Liu C, Karin M and Edgington TS: A large noncoding RNA is a marker for murine hepatocellular carcinomas and a spectrum of human carcinomas. Oncogene 26: 851-858, 2007.

11. Calin GA, Liu CG, Ferracin M, et al: Ultraconserved regions encoding ncRNAs are altered in human leukemias and carcinomas. Cancer Cell 12: 215-229, 2007.

12. Braconi C, Valeri N, Kogure T, et al: Expression and functional role of a transcribed noncoding RNA with an ultraconserved element in hepatocellular carcinoma. Proc Natl Acad Sci USA 108: 786-791, 2011.

13. Gibb EA, Brown CJ and Lam WL: The functional role of long non-coding RNA in human carcinomas. Molecular Cancer 10: 38, 2011.

14. Qi P and Du X: The long non-coding RNAs, a new cancer diagnostic and therapeutic gold mine. Mod Pathol 26: 155-165, 2013. 
15. Weakley SM, Wang H, Yao Q and Chen C: Expression and function of a large non-coding RNA gene XIST in human cancer. World J Surg 35: 1751-1756, 2011.

16. Wang J, Liu X, Wu H, et al: CREB up-regulates long non-coding RNA, HULC expression through interaction with microRNA372 in liver cancer. Nucleic Acids Res 38: 5366-5383, 2010.

17. Yuan S-X, Yang F, Yang Y, et al: Long noncoding RNA associated with microvascular invasion in hepatocellular carcinoma promotes angiogenesis and serves as a predictor for hepatocellular carcinoma patients' poor recurrence-free survival after hepatectomy. Hepatology 56: 2231-2241, 2012.

18. Yang F, Xue X, Bi J, et al: Long noncoding RNA CCAT1, which could be activated by c-Myc, promotes the progression of gastric carcinoma. J Cancer Res Clin Oncol 139: 437-445, 2013.

19. Wu W, Bhagat TD, Yang X, et al: Hypomethylation of noncoding DNA regions and overexpression of the long noncoding RNA, $A F A P 1-A S 1$, in Barrett's esophagus and esophageal adenocarcinoma. Gastroenterology 144: 956-966, 2013

20. Tahira AC, Kubrusly MS, Faria MF, et al: Long noncoding intronic RNAs are differentially expressed in primary and metastatic pancreatic cancer. Mol Cancer 10: 141, 2011.

21. Merchant JL: Inflammation, atrophy, gastric cancer: connecting the molecular dots. Gastroenterology 129: 1079-1082, 2005.

22. Huarte M, Guttman M, Feldser D, et al: A large intergenic noncoding RNA induced by 553 mediates global gene repression in the p53 response. Cell 142: 409-419, 2010.

23. Zhai H, Fesler A, Schee K, Fodstad Ø, Flatmark K and Ju J: Clinical significance of long intergenic noncoding RNA-p21 in colorectal cancer. Clin Colorectal Cancer 12: 261-266, 2013.

24. Yoon JH, Abdelmohsen K, Srikantan S, et al: LincRNA-p21 suppresses target mRNA translation. Mol Cell 47: 648-655, 2012

25. Samuels Y and Velculescu VE: Oncogenic mutations of PIK3CA in human cancers. Cell Cycle 3: 1221-1224, 2004.

26. Wu K, Yang Q, Mu Y,Zhou L, Liu Y,Zhou Q and He B: Berberine inhibits the proliferation of colon cancer cells by inactivating Wnt//3-catenin signaling. Int J Oncol 41: 292-298, 2012.

27. Dihlmann S, Klein S and Doeberitz Mv Mv: Reduction of $\beta$-catenin/T-cell transcription factor signaling by aspirin and indomethacin is caused by an increased stabilization of phosphorylated $\beta$-catenin. Mol Cancer Ther 2: 509-516, 2003.

28. Boon EM, Keller JJ, Wormhoudt TA, et al: Sulindac targets nuclear $\beta$-catenin accumulation and Wnt signalling in adenomas of patients with familial adenomatous polyposis and in human colorectal cancer cell lines. Br J Cancer 90: 224-229, 2004.

29. Ji BC, Hsu WH, Yang JS, et al: Gallic acid induces apoptosis via caspase-3 and mitochondrion-dependent pathways in vitro and suppresses lung xenograft tumor growth in vivo. J Agric Food Chem 57: 7596-7604, 2009.

30. Xi Y, Nakajima G, Gavin E, et al: Systematic analysis of microRNA expression of RNA extracted from fresh frozen and formalin-fixed paraffin-embedded samples. RNA 13: 1668-1674, 2007.

31. Wang K, Zhang R, He F, et al: Electroacupuncture frequencyrelated transcriptional response in rat arcuate nucleus revealed region-distinctive changes in response to low- and high-frequency electroacupuncture. J Neurosci Res 90: 1464-1473, 2012.

32. Kalin TV, Wang IC, Ackerson TJ, et al: Increased levels of the FoxM1 transcription factor accelerate development and progression of prostate carcinomas in both TRAMP and LADY transgenic mice. Cancer Res 66: 1712-1720, 2006.
33. Kim IM, Ackerson T, Ramakrishna S, et al: The Forkhead Box $\mathrm{m} 1$ transcription factor stimulates the proliferation of tumor cells during development of lung cancer. Cancer Res 66: 2153-2161, 2006

34. Glimelius B and Oliveira J; ESMO Guidelines Working Group Rectal cancer: ESMO clinical recommendations for diagnosis, treatment and follow-up. Ann Oncol 20 (Suppl 4): S54-S56, 2009.

35. Sauer R, Becker H, Hohenberger W, et al: Preoperative versus postoperative chemoradiotherapy for rectal cancer. N Engl J Med 351: 1731-1740, 2004.

36. Koukourakis MI, Giatromanolaki A, Sheldon $\mathrm{H}$, et al: Phase I/II trial of bevacizumab and radiotherapy for locally advanced inoperable colorectal cancer: vasculature-independent radiosensitizing effect of bevacizumab. Clin Cancer Res 15: 7069-7076, 2009.

37. Kendziorra E, Ahlborn K, Spitzner M, et al: Silencing of the Wnt transcription factor TCF4 sensitizes colorectal cancer cells to (chemo-) radiotherapy. Carcinogenesis 32: 1824-1831, 2011.

38. Özgür E, Mert U, Isin M, Okutan M, Dalay N and Gezer U: Differential expression of long non-coding RNAs during genotoxic stress-induced apoptosis in HeLa and MCF-7 cells. Clin Exp Med 13: 119-126, 2013.

39. Jemal A, Center MM, Ward E and Thun MJ: Cancer occurrence. Methods Mol Biol 471: 3-29, 2009.

40. Bordonaro M: Crosstalk between Wnt signaling and RNA processing in colorectal cancer. J Cancer 4: 96-103, 2013.

41. Li X, Pu J, Jiang S, et al: Henryin, an ent-kaurane diterpenoid, inhibits Wnt signaling through interference with $\beta$-catenin/TCF4 interaction in colorectal cancer cells. PLoS One 8: e68525, 2013.

42. Waaler J, Machon O, von Kries JP, et al: Novel synthetic antagonists of canonical Wnt signaling inhibit colorectal cancer cell growth. Cancer Res 71: 197-205, 2011.

43. Bright-Thomas RM and Hargest R: APC, $\beta$-Catenin and hTCF-4; an unholy trinity in the genesis of colorectal cancer. Eur J Surg Oncol 29: 107-117, 2003.

44. He TC, Sparks AB, Rago C, et al: Identification of c-MYC as a target of the APC pathway. Science 281: 1509-1512, 1998

45. Khor TO, Gul YA, Ithnin H and Seow HF: A comparative study of the expression of Wnt-1, WISP-1, survivin and cyclin-D1 in colorectal carcinoma. Int J Colorectal Dis 21: 291-300, 2006.

46. Cunningham D, Atkin W, Lenz HJ, et al: Colorectal cancer. Lancet 375: 1030-1047, 2010.

47. Raats DA, de Bruijn MT, Steller EJ, Emmink BL, Borel-Rinkes IH and Kranenburg O: Synergistic killing of colorectal cancer cells by oxaliplatin and ABT-737. Cell Oncol (Dordr) 34: 307-313, 2011.

48. Shibue T, Takeda K, Oda E, et al: Integral role of Noxa in p53-mediated apoptotic response. Genes Dev 17: 2233-2238, 2003.

49. Miyoshi-Imamura T, Kakinuma S, Kaminishi M, et al: Unique characteristics of radiation-induced apoptosis in the postnatally developing small intestine and colon of mice. Radiat Res 173: 310-318, 2010. 